
\title{
$\angle S$ Research Suare \\ The Effect of Guilu Erxian Jiao on Osteoporosis Patients: A Retrospective Study
}

\section{Yuan-Xin Lai ( $\nabla$ u9802401@cmu.edu.tw)}

Chang Gung Memorial Hospital https://orcid.org/0000-0001-7305-0496

\section{Chu-Yao Tseng}

Chang Gung Memorial Hospital Taoyuan Branch: Taoyuan Chang Gung Memorial Hospital

\section{Huang-Yu Yang}

Chang Gung Kidney Research Center

\section{Sien-Hung Yang}

Chang Gung Memorial Hospital Taipei Branch: Chang Gung Memorial Hospital

\section{Tsung-Hsien Yang}

New Taipei City Municipal Tucheng Hospital

\section{Research}

Keywords: Chinese herbal medicine, Guilu Erxian Jiao, osteoporosis, T-score, fracture

Posted Date: July 13th, 2021

DOI: https://doi.org/10.21203/rs.3.rs-685272/v1

License: (c) (i) This work is licensed under a Creative Commons Attribution 4.0 International License. Read Full License 


\section{Abstract}

Background: This retrospective study aimed to evaluate whether the Chinese herbal medicine formula Guilu Erxian Jiao can improve T-score in osteoporosis patients diagnosed by dual energy x-ray absorptiometry (DXA) and even decrease fracture rate.

Method: Osteoporosis participants were collected through the Chang Gung Memorial Hospital (CGMH) database from 2000 to 2019. Intervention was taking Guilu Erxian Jiao pills. We analyzed the change of T-score and the following cumulative incidence of fracture. The Student's T-test, Cox's proportional hazard model, Kaplan-Meier method and log-rank test were conducted using SAS software.

Results: There was no significant difference about the baseline between the Guilu Erxian Jiao group and non-Guilu Erxian Jiao group. Guilu Erxian Jiao can improve the osteoporosis of the vertebrate of lumbar. However, there is no significant diffidence about the vertebrate of lumbar and femoral neck between the Guilu Erxian Jiao group and non-Guilu Erxian Jiao group. The cumulative rate of fracture between these two groups is no significant difference. However, if patients took the total dose of Guilu Erxian Jiao over 600 pill*days, the fracture rate would decrease.

Conclusion: Usage of Guilu Erxian Jiao would improve the osteoporosis of vertebrate of lumbar, but does not improve the cumulative incidence of fracture, unless they took the total dose of Guilu Erxian Jiao over 600 pill*days.

\section{Introduction}

Osteoporosis is a life threatening problem worldwide, and even in highly developed country United States at least 10 millions of people have osteoporosis [1]'[2]. People having osteoporosis would experience hip fracture sooner, which is a mortal event to elderly[3]. The first-time of hip fracture can lead $30 \%$ death rate in one year [4]. Osteoporosis can be divided into two group primary osteoporosis and secondary osteoporosis. Primary osteoporosis includes postmenopausal women and aged-over 65-yeay-old men. Besides, low body-mass index, previous fragility fractures, a family history of fractures, the use of glucocorticoids, active cigarette smoking, and alcohol drinking are all risk factors of osteoporosis [5]. The medicine treatment of osteoporosis includes two categories, anabolic drugs, stimulating bone formation and antiresorptive drugs, slowing down bone resorption. It takes at least one year to achieve the goal of decrease fracture rate. It is suggested that bone mineral density (BMD) assessed with dual x-ray absorptiometry (DXA) should be followed every two year in osteoporosis patients [6] .

With fewer side effects, Chinese herbal medicines (CHMs) have been one of the mainstream therapies in Asian countries [7]. Based on the traditional Chinese medicine (TCM) theory, osteoporosis is believed to occur due to the deficiency of Shen (kidney) essence, which plays a decisive role in nourishing the bone and strengthening the skeleton [8]. Guilu Erxian Jiao is a nourishment Chinese herbal medicine, which is labeled to treat osteoporosis and is paid by National Health Insurance in Taiwan [9]. In an animal study, Guilu Erxian Jiao can promote osteoblastic differentiation marker ALP activity and increase bone 
morphogenetic protein - 2 production, which is a key regulator in bone formation and osteoblastic differentiation [10]. In a clinical trial, Guilu Erxian Jiao increased mean BMD of postmenopausal women especially on lumbar spine and femoral neck by 12 weeks intake, significantly greater than before [11].

This study is a retrospective study to assess the effect after taking Guilu Erxian Jiao in Chang Gung Memorial Hospital (CGMH). We hope to evaluate these patients who were diagnosed as osteoporosis by DXA (T-score $\leq-2.5$ ) [12], and we analyze their use of osteoporosis drugs and/or Guilu Erxian Jiao after the first DXA and before fractures. Besides, we hope to assess the change of T-score and the occurrence of hip fractures or lumbar compression fractures in patients who have used osteoporosis drugs and/or Guilu Erxian Jiao.

\section{Materials And Methods}

Data source

The subjects of this cohort study were retrieved from CGMH database. Through CGMH database, we collected related data from the Taipei, Linkou, Taoyuan, Keelung, Yunlin, Chiayi and Kaohsiung districts of the hospital from January 1,2000, to December 31, 2019. The CGMH database provides anonymized and encrypted data of patients for research purposes.

Study population

Our population cohort study used included 1.DXA examination twice at least during 2000 to 2019, and 2.the period between the twice DXA examination was at interval at least three months but no more than two years (Fig. 1).3. Subjects were required age above eighteen full years old. The exclusion criteria included: 1. patients who received Guilu Erxian Jiao treatment more than 90 days before the first-time DXA examination, 2. the age less than 18 years old, 3. incomplete information of data, and 4.T-score>-2.5.

Approval for the study was obtained from the Chang Gung Medical Foundation Institutional Review Board.

The background characteristics of the subjects were described in Table 1. Comorbidity diseases included cancer, cardiovascular disease, chronic kidney disease, chronic obstructive pulmonary disease, dementia, diabetes mellitus, depression, hypertension, Parkinson's disease, hyperparathyroidism, multiple myeloma, and rheumatic arthritis. Medication for osteoporosis were bisphosphates, RANKL inhibitors, sex hormones, calcitriol, Teriparatide, and steroids. Osteoporosis were defined as T-score $\leq-2.5$ [12]. 
Table 1

Demographic data and selected comorbidities of the study subjects

\begin{tabular}{|c|c|c|c|c|c|}
\hline \multirow[t]{3}{*}{ Variables } & \multicolumn{2}{|c|}{$\begin{array}{l}\text { Non-Guilu Erxian Jiao } \\
\text { group }\end{array}$} & \multicolumn{3}{|c|}{$\begin{array}{l}\text { Guilu Erxian Jiao } \\
\text { group }\end{array}$} \\
\hline & \multicolumn{2}{|c|}{$n=425$} & \multicolumn{2}{|l|}{$\mathrm{n}=85$} & \multirow[t]{2}{*}{$\mathrm{p}$-value } \\
\hline & mean & SD & mean & SD & \\
\hline age at 1 st DXA(year) & 62.35 & 12.20 & 61.05 & 12.87 & 0.375 \\
\hline \multirow[t]{2}{*}{ Interval between 2 DXA } & 14.65 & 5.28 & 14.86 & 5.04 & 0.733 \\
\hline & $\mathrm{n}$ & $\%$ & $\mathrm{n}$ & $\%$ & \\
\hline \multicolumn{6}{|l|}{ age } \\
\hline age $<65$ & 280 & $65.88 \%$ & 56 & $65.88 \%$ & 1.000 \\
\hline age $>=65$ & 145 & $34.12 \%$ & 29 & $34.12 \%$ & \\
\hline \multicolumn{6}{|l|}{ gender } \\
\hline male & 70 & $16.47 \%$ & 14 & $16.47 \%$ & 1.000 \\
\hline female & 355 & $83.53 \%$ & 71 & $83.53 \%$ & \\
\hline \multicolumn{6}{|l|}{ Fracture before 1st DXA } \\
\hline Femoral neck fracture before & 2 & $0.47 \%$ & 0 & $0.00 \%$ & 1.000 \\
\hline Lumbar vertebra Fracture before & 17 & $4.00 \%$ & 5 & $5.88 \%$ & 0.390 \\
\hline Thoracic vertebra Fracture before & 9 & $2.12 \%$ & 1 & $1.18 \%$ & 1.000 \\
\hline Radius ulna Fracture before & 7 & $1.65 \%$ & 1 & $1.18 \%$ & 1.000 \\
\hline $\begin{array}{l}\text { Proximal humerus Fracture } \\
\text { before }\end{array}$ & 3 & $0.71 \%$ & 0 & $0.00 \%$ & 1.000 \\
\hline \multicolumn{6}{|l|}{ Comorbidity } \\
\hline Cancer & 88 & $20.71 \%$ & 16 & $18.82 \%$ & 0.806 \\
\hline Cardiovascular disease & 57 & $13.41 \%$ & 16 & $18.82 \%$ & 0.258 \\
\hline Chronic kidney disease & 22 & $5.18 \%$ & 0 & $0.00 \%$ & 0.035 \\
\hline COPD & 80 & $18.82 \%$ & 13 & $15.29 \%$ & 0.538 \\
\hline Dementia & 6 & $1.41 \%$ & 3 & $3.53 \%$ & 0.177 \\
\hline Diabetes Mellitus & 59 & $13.88 \%$ & 6 & $7.06 \%$ & 0.123 \\
\hline Depression & 22 & $5.18 \%$ & 6 & $7.06 \%$ & 0.442 \\
\hline Hypertension & 121 & $28.47 \%$ & 23 & $27.06 \%$ & 0.895 \\
\hline
\end{tabular}




\begin{tabular}{|llllll|}
\hline Variables & \multicolumn{3}{l}{$\begin{array}{l}\text { Non-Guilu Erxian Jiao } \\
\text { group }\end{array}$} & \multicolumn{3}{l|}{$\begin{array}{l}\text { Guilu Erxian Jiao } \\
\text { group }\end{array}$} \\
\hline Parkinson's disease & 2 & $0.47 \%$ & 2 & $2.35 \%$ & 0.131 \\
\hline Hyperparathyroidism & 4 & $0.94 \%$ & 2 & $2.35 \%$ & 0.263 \\
\hline Multiple myeloma & 1 & $0.24 \%$ & 0 & $0.00 \%$ & 1.000 \\
\hline Rheumatic arthritis & 17 & $4.00 \%$ & 2 & $2.35 \%$ & 0.753 \\
\hline Medication for Osteoporosis & & & & & \\
\hline Bisphosphates & 87 & $20.47 \%$ & 15 & $17.65 \%$ & 0.656 \\
\hline RANKL inhibitors & 81 & $19.06 \%$ & 15 & $17.65 \%$ & 0.879 \\
\hline Sex Hormones & 80 & $18.82 \%$ & 11 & $12.94 \%$ & 0.255 \\
\hline Calcitriol & 20 & $4.71 \%$ & 3 & $3.53 \%$ & 0.781 \\
\hline Teriparatide & 16 & $3.76 \%$ & 3 & $3.53 \%$ & 1.000 \\
\hline Steroid & 43 & $10.12 \%$ & 8 & $9.41 \%$ & 1.000 \\
\hline
\end{tabular}

Study design

Patients who received Guilu Erxian Jiao treatment and without Guilu Erxian Jiao treatment were divided into study group and control group in a 1:5 matching, which made no significant difference in basic values (Table 1).

Assessment

We assessed the T-score and fracture rate to answer whether osteoporosis patients taking Guilu Erxian Jiao treatment could increase bone mineral density or fracture rate and how much dose we should take to improve fracture rate.

\section{Statistical analysis}

Categorical variables were reported as numbers. The difference in proportions and T-score was assessed using the Student's T-test as appropriate. Cox's proportional hazard model estimated hazard ratios (HR) of Guilu Erxian Jiao usage on fractures rate. The difference in fracture development between the two groups was estimated using the Kaplan-Meier method and the log-rank test. The database software, SAS Enterprise Guide version 9.4 (SAS Institute Inc., Cary, NC, USA), was used for data processing and analysis at a $p<0.05$ statistically significant level.

\section{Results}


There were 218 Guilu Erxian Jiao users and 19879 non-Guilu Erxian Jiao users among the twice DXA examination patients. After exclusion criteria, there were 85 osteoporosis patients in the Guilu Erxian Jiao group. After matching procedure with propensity score, there were 425 osteoporosis patients in the nonGuilu Erxian Jiao group. There was no significant difference between the two groups in age, sex, interval between twice DXA, fracture before 1st DXA, comorbidity, and medication usage for osteoporosis, indicating the two groups to be comparable on these characteristics (Table 1). The fracture rate after 1st DXA was significantly different in lumbar spine $(P=0.034)$,but not different in femoral neck, thoracic vertebrae, radius ulna and proximal humerus (Table 2).

Table 2

Fracture rate after first DXA in Guilu Erxian Jiao group and non-Guilu Erxian Jiao group.

\begin{tabular}{|llllll|}
\hline $\begin{array}{l}\text { Fracture after 1st DXA(new } \\
\text { case) }\end{array}$ & \multicolumn{2}{l}{$\begin{array}{l}\text { Non-Guilu Erxian Jiao } \\
\text { group }\end{array}$} & \multicolumn{3}{l|}{ Guilu Erxian Jiao group } \\
\hline Femoral neck fracture after & $\mathrm{n}$ & $\%$ & $\mathrm{n}$ & $\%$ & $\mathrm{p}$-value \\
\hline Lumbar vertebrae fracture after & 58 & $13.65 \%$ & 4 & $4.71 \%$ & 1.000 \\
\hline Thoracic vertebrae fracture after & 20 & $4.71 \%$ & 5 & $5.88 \%$ & 0.034 \\
\hline Radius ulna fracture after & 14 & $3.29 \%$ & 3 & $3.53 \%$ & 1.000 \\
\hline Proximal humerus fracture after & 3 & $0.71 \%$ & 1 & $1.18 \%$ & 0.519 \\
\hline $\mathrm{p}<0.05$ were bold. & & & & & \\
\hline
\end{tabular}

Comparison of Guilu Erxian Jiao group with non-Guilu Erxian Jiao group, there was no significant difference in T-scores of lumbar spine and left femoral neck. The data suggested that the T-score of the left hip was significantly different with the non-Guilu Erxian Jiao group compared with the Guilu Erxian Jiao group (mean=-2.3, $p=0.036$; Table 3), which indicated BMD of left hip in non-Guilu Erxian Jiao group appeared more severe since 1st DXA examination. 
Table 3

T-score of lumbar, hip, and femoral neck before and after examination comparied between Guilu Erxian Jiao use and no-use gruop among osteoporosis patients

\begin{tabular}{|llllll|}
\hline \multicolumn{5}{|c|}{ Non-Guilu Erxian Jiao group } & \multicolumn{2}{l|}{ Guilu Erxian Jiao group } \\
\hline & $\mathrm{n}=425$ & & $\mathrm{n}=85$ & $\mathrm{p}$-value \\
\hline \multicolumn{1}{|c|}{ mean } & SD & mean & SD & \\
\hline Lumbar & & & & \\
\hline T-score before & -2.77 & 1.08 & -2.61 & 1.03 & 0.235 \\
\hline T-score after & -2.56 & 1.13 & -2.42 & 1.04 & 0.298 \\
\hline L Hip & & & & & \\
\hline T-score before & -2.51 & 0.81 & -2.30 & 0.84 & 0.036 \\
\hline T-score after & -2.43 & 0.86 & -2.19 & 0.89 & 0.024 \\
\hline L_Fneck & & & & & 0.124 \\
\hline T-score before & -3.08 & 0.86 & -2.88 & 1.14 & 0.057 \\
\hline T-score after & -2.98 & 0.88 & -2.78 & 0.83 & \\
\hline L, left; F, femoral. & & & & \\
\hline P < 0.05 were bold. & & & & \\
\hline
\end{tabular}

In the Guilu Erxian Jiao group, there was significant difference in lumbar spine T-score $(p<0.001$; Table 4), and the increase in left hip T-scores appeared to be strongly associated with the use of Guilu Erxian Jiao ( $P=0.0117)$. However, there was no significant difference in left femoral neck. In contrast, non-Guilu Erxian Jiao group showed significant difference in lumbar spine, left hip and left femoral $\operatorname{neck}(P<0.0001 \square P<0.0001 \square P=0.0002 ;$ Table 4). 
Table 4

T-score before and after Guilu Erxian Jiao use/no use intra-group comparison

\begin{tabular}{|c|c|c|c|c|c|c|}
\hline & \multicolumn{3}{|c|}{$\begin{array}{l}\text { Non-Guilu Erxian Jiao } \\
\text { group }\end{array}$} & \multicolumn{3}{|c|}{$\begin{array}{l}\text { Guilu Erxian Jiao } \\
\text { group }\end{array}$} \\
\hline & \multicolumn{2}{|l|}{$n=425$} & \multirow[t]{2}{*}{ p-value } & \multicolumn{2}{|l|}{$\mathrm{n}=85$} & \multirow{2}{*}{$\begin{array}{l}\mathrm{p}- \\
\text { value }\end{array}$} \\
\hline & mean & sd & & mean & $s d$ & \\
\hline \multicolumn{7}{|l|}{ Lumbar } \\
\hline $\begin{array}{l}\text { T-score before vs T-score } \\
\text { after }\end{array}$ & 0.21 & 0.58 & $\hat{0} .0001$ & 0.19 & 0.46 & 0.0002 \\
\hline \multicolumn{7}{|l|}{ L_Hip } \\
\hline $\begin{array}{l}\text { T-score before vs T-score } \\
\text { after }\end{array}$ & 0.08 & 0.39 & $\hat{0} .0001$ & 0.11 & 0.39 & 0.0117 \\
\hline \multicolumn{7}{|l|}{ L_Femoral neck } \\
\hline $\begin{array}{l}\text { T-score before vs T-score } \\
\text { after }\end{array}$ & 0.10 & 0.56 & 0.0002 & 0.08 & 1.09 & 0.4839 \\
\hline$p<0.05$ were bold. & & & & & & \\
\hline
\end{tabular}

Table 5 shows the risk of fracture occurrence in the Guilu Erxian Jiao group and non-Guilu Erxian Jiao group, in which there was no significant difference, except bisphosphates use group $(p=0.0012 ;$ Table5). The bisphosphates use group experienced fracture before 1st DXA more than others group, which had significant difference $(p<0.001$; Table 6$)$. 
Table 5

The fracture rate after medication use and/or Guilu Erxian Jiao use.

\begin{tabular}{|llll|}
\hline \multicolumn{4}{|c|}{ Univariate cox } \\
\hline & HR & $95 \% \mathrm{Cl}$ & p-value \\
\hline group & & & \\
\hline Guilu Erxian Jiao group(A) & 0.70 & $0.33-1.46$ & 0.338 \\
\hline Non-Guilu Erxian group (B) & ref & & \\
\hline Medication for Osteoporosis & & & \\
\hline Bisphosphates & 1.94 & $1.16-3.24$ & 0.012 \\
\hline RANKL inhibitors & 1.34 & $0.75-2.38$ & 0.323 \\
\hline Sex hormones & 0.84 & $0.44-1.61$ & 0.603 \\
\hline Calcitriol & 0.61 & $0.15-2.48$ & 0.486 \\
\hline Teriparatide & 2.26 & $0.91-5.61$ & 0.08 \\
\hline Steroid & 1.16 & $0.56-2.43$ & 0.689 \\
\hline
\end{tabular}

Table 6

the experience of fracture before first DXA with/or without Bisphosphates

\begin{tabular}{|c|c|c|c|c|c|}
\hline \multicolumn{6}{|l|}{ Bisphosphates } \\
\hline & \multicolumn{2}{|c|}{ Non-Bisphosphates use } & \multicolumn{2}{|c|}{ Bisphosphates use } & \multirow[t]{2}{*}{ P-value } \\
\hline & $\mathrm{N}$ & $\%$ & $\mathrm{~N}$ & $\%$ & \\
\hline No fracture before 1st DXA & 390 & 95.59 & 82 & 80.39 & $<0.0001$ \\
\hline Fracture before 1st DXA & 18 & 4.41 & 20 & 19.61 & \\
\hline
\end{tabular}

Based on Kaplan-Meier survival curve and log-rank test results, the data also showed no statistically significant difference regarding the survival rate of free from fracture across the groups during the followup five years period. Those receiving Guilu Erxian Jiao did not have significantly lower incidence rate of fracture than those not receiving Guilu Erxian Jiao ( $p=0.335$; Fig. 2).

Another Kaplan-Meier curve of the difference between the non-medicine and non-Guilu Erxian Jiao group, the medicine and non-Guilu Erxian Jiao group, non-medicine plus Guilu Erxian Jiao group, and medicine plus Guilu Erxian Jiao group also showed no significant difference in incidence rate of fracture (Fig. 3). 
The receiver operating characteristic curve (ROC curve) represented the total amount of 600 pills of Guilu Erxian Jiao to be an ideal discrimination point(Fig. 4). There was significant difference between the high total dose Guilu Erxian Jiao group, and the low total dose Guilu Erxian Jiao group in the development of fracture ( $=0.0039$; Fig. 5).

\section{Discussion}

It is important that the prevention of osteoporosis prevalence and incidence due to the aging society in developing countries [13],[14]. Many researches had emphasis on the importance of traditional Chinese medicine to osteoporosis and fracture $[9] \cdot[15,16]$. Among them, Guilu Erxian Jiao was often prescribed [17]'[18]. In a clinical research, postmenopausal women(mean age 54.8 [19]'[20])had improve on BMD after three months treatment at the lumbar spine [11].It was a pity that most data of those researches were evaluated through $\mathrm{NHI}$ database, which meant the lack of following reports. That was why our cohort study set twice DXA examination record as the first criteria to include osteoporosis patients and to observe the effect of Guilu Erxian Jiao.

Guilu Erxian Jiao was the mostly prescribed formula in $\mathrm{CGMH}$, and the number of osteoporosis patient taking twice DXA was over 40000 . However, the number of patients who used Guilu Erxian Jiao and took twice DXA was only 218.The reason might be Guilu Erxian Jiao prescribed in CGMH but the DXA examination done in other hospitals, causing the loss of clinical data. On one hand, the data was anonymized and delinked. On the other hand, the DXA reports form and contents changed owing to different radiologist and different hospital distriction. Those all diminished our case number.

Compared with the previous Taiwan NHI database study, they demonstrated TCM use can decrease the rate of osteoporotic fracture rate, in this studty, Guilu Erxian Jiao is the mainly prescribed formula [9]. However, our study did not achieve this result. The reason might be owing to the large difference of total amounts of Guilu Erxian Jiao patents taking in our retrospective study.

Although our study results did not meet our expectation, we found Guilu Erxian Jiao could improve osteoporosis in the lumbar spine, and BMD in the hip joint, which played an important role in the prevention of hip fracture [4] [21]. A previous animal study also shows Guilu Erxian Jiao can treat resorptive disease such as osteoporosis by inhibiting osteoclasts resorption [22]. This result is compatible for our study.

We also demonstrated that patients who took high total Guilu Erxian Jiao dose at least 600 pill*days had a lower incidence of fracture rate. This result may be a guide for future research.

We also found there was higher fracture rate in osteoporosis patients taking bisphosphates. It was owing to the low T-score in this group whom needed take bisphosphates. Therefore, this group had higher fracture rate in the following study. 
Besides, the reason why non-medicine and non-Guilu Erxian Jiao group seemed to be less fracture rate was attributed to the severity of osteoporosis, which means they did not took medicine due to the better performance of their bone mass.

This study has several limitations. First, data associated Guilu Erxian Jiao use were small, as such data cannot be obtained from other hospitals. This restricted the group matching to1:5. Second, investigations of ethical, psychological, and cultural factors were limited due to the nature of the database, which restricted investigations of rural and urban diversities.

\section{Conclusion}

This is the first large-scale cohort study to investigate the effect of Guilu Erxian Jiao on the risk of osteoporosis and fracture rates among osteoporosis patients. This study suggested usage of Guilu Erxian Jiao would improve the osteoporosis of vertebrate of lumbar, but not improve the cumulative incidence of fracture. However, if patients took the total dose of Guilu Erxian Jiao over 600 pill*days, the fracture rate will decrease.

\section{Abbreviations}

DXA: dual energy x-ray absorptiometry; CGMH: Chang Gung Memorial Hospital; BMD: bone mineral density; CHMs: Chinese herbal medicines; TCM: traditional Chinese medicine

\section{Declarations}

\section{Author Contribution}

Yuan-Xin Lai and Tsung-Hsien Yang designed the experiments, performed data analysis and statistics, and drafted the manuscript. Chu-yao Tseng participated in the study design and helped with the data analysis. Yang Huang-Yu and Sien-Hung Yang provided professional comments for this work. All authors read and approved the final manuscript.

Author details

${ }^{1}$ Division of Chinese Acupuncture and Traumatology, Center of Traditional Chinese Medicine, Chang Gung Memorial Hospital Taipei Branch, Taipei, Taiwan

${ }^{2}$ Division of Chinese Acupuncture and Traumatology, Center of Traditional Chinese Medicine, Chang Gung Memorial Hospital, Taoyuan, Taiwan

${ }^{3}$ Kidney Research Center, Department of Nephrology, Chang Gung Immunology Consortium, Chang Gung Memorial Hospital, Chang Gung University College of Medicine, Taoyuan , Taiwan 
${ }^{4}$ Department of Traditional Chinese Medicine, Division of Chinese Internal Medicine, Chang Gung Memorial Hospital Taipei Branch, Taipei, Taiwan

${ }^{5}$ School of Chinese Medicine, Chang Gung University, Taoyuan, Taiwan

${ }^{6}$ Research Center for Chinese Herbal Medicine,Chang Gung University of Science and Technology, Taoyuan, Taiwan

${ }^{7}$ Department of Traditional Chinese Medicine, New Taipei Municipal TuCheng Hospital, New Taipei, Taiwan

Acknowledgements

This work was supported by grants from Chang Gung Memorial Hospital (CORPG3H0361). The authors would like to thank the Center for Big data Analytics and Statistics, Chang Gung Memorial Hospital, Linkou for statistical assistance and consultation and data analysis.

Competing interests

The authors declare that they have no competing interests.

Availability of data and materials

Please contact author for data requests.

Ethics approval and consent to participate

Prior to commencement of the study, ethical approval (201800780B0C501) (Additional file 1) was obtained from the Chang Gung Medical Foundation Institutional Review Board.

Consent to publish

The authors give full consent to publish.

\section{References}

1. Srivastava M, Deal C. Osteoporosis in elderly: prevention and treatment. Clinics in Geriatric Medicine. 2002;18:529-55. doi: https://doi.org/10.1016/S0749-0690(02)00022-8.

2. Osteoporosis prevention, diagnosis, and therapy. Jama. 2001;285:785-95. doi: 10.1001/jama.285.6.785.

3. Lewiecki EM, Wright NC, Curtis JR, Siris E, Gagel RF, Saag KG, et al. Hip fracture trends in the United States, 2002 to 2015. Osteoporos Int. 2018;29:717-22. doi: 10.1007/s00198-017-4345-0.

4. Lee SH, Chen IJ, Li YH, Fan Chiang $\mathrm{CY}$, Chang $\mathrm{CH}$, Hsieh PH. Incidence of second hip fractures and associated mortality in Taiwan: A nationwide population-based study of 95,484 patients during 
2006-2010. Acta Orthop Traumatol Turc. 2016;50:437-42. doi: 10.1016/j.aott.2016.06.008.

5. Lane NE. Epidemiology, etiology, and diagnosis of osteoporosis. Am J Obstet Gynecol. 2006;194:S311. doi: 10.1016/j.ajog.2005.08.047.

6. Rachner TD, Khosla S, Hofbauer LC. Osteoporosis: now and the future. The Lancet. 2011;377:127687. doi: 10.1016/s0140-6736(10)62349-5.

7. Zhou X, Li CG, Chang D, Bensoussan A. Current Status and Major Challenges to the Safety and Efficacy Presented by Chinese Herbal Medicine. Medicines (Basel). 2019;6. doi: 10.3390/medicines6010014.

8. Liu Y, Liu JP, Xia Y. Chinese herbal medicines for treating osteoporosis. Cochrane Database Syst Rev. 2014:CD005467. doi: 10.1002/14651858.CD005467.pub2.

9. Wang YC, Chiang JH, Hsu HC, Tsai $\mathrm{CH}$. Decreased fracture incidence with traditional Chinese medicine therapy in patients with osteoporosis: a nationwide population-based cohort study. BMC Complement Altern Med. 2019;19:42. doi: 10.1186/s12906-019-2446-3.

10. Wu MH, Lee TH, Lee HP, Li TM, Lee IT, Shieh PC, et al. Kuei-Lu-Er-Xian-Jiao extract enhances BMP-2 production in osteoblasts. Biomedicine (Taipei). 2017;7:2. doi: 10.1051/bmdcn/2017070102.

11. Li R-S, Shyu J-Y, Sun S-Y, Ho F-M, Wang S-H. The Effect of Guilu Erxian Decoction on Bone Mineral Density in Postmenopausal Women Evaluated Using Dual Energy X-ray Absorptiometry and Quantitative Ultrasound. C J Radiologic Tech. 2007;31:147-53. doi: 10.30045/cjrt.200712.0009.

12. Sozen T, Ozisik L, Basaran NC. An overview and management of osteoporosis. Eur J Rheumatol. 2017;4:46-56. doi: 10.5152/eurjrheum.2016.048.

13. Aziziyeh R, Amin M, Habib M, Garcia Perlaza J, Szafranski K, McTavish RK, et al. The burden of osteoporosis in four Latin American countries: Brazil, Mexico, Colombia, and Argentina. J Med Econ. 2019;22:638-44. doi: 10.1080/13696998.2019.1590843.

14. Burge R, Dawson-Hughes B, Solomon DH, Wong JB, King A, Tosteson A. Incidence and economic burden of osteoporosis-related fractures in the United States, 2005-2025. J Bone Miner Res. 2007;22:465-75. doi: 10.1359/jbmr.061113.

15. Jin YX, Wu P, Mao YF, Wang B, Zhang JF, Chen WL, et al. Chinese Herbal Medicine for Osteoporosis: A Meta-analysis of Randomized Controlled Trials. J Clin Densitom. 2017;20:516-25. doi: 10.1016/j.jocd.2017.07.003.

16. Lee H, Kim MH, Choi Y, Yang WM. Ameliorative effects of Osteo-F, a newly developed herbal formula, on osteoporosis via activation of bone formation. J Ethnopharmacol. 2021;268:113590. doi: 10.1016/j.jep.2020.113590.

17. Shih WT, Yang YH, Chen PC. Prescription patterns of chinese herbal products for osteoporosis in taiwan: a population-based study. Evid Based Complement Alternat Med. 2012;2012:752837. doi: $10.1155 / 2012 / 752837$.

18. Zhang ND, Han T, Huang BK, Rahman K, Jiang YP, Xu HT, et al. Traditional Chinese medicine formulas for the treatment of osteoporosis: Implication for antiosteoporotic drug discovery. $\mathrm{J}$ Ethnopharmacol. 2016;189:61-80. doi: 10.1016/j.jep.2016.05.025. 
19. Shen TY, Strong C, Yu T. Age at menopause and mortality in Taiwan: A cohort analysis. Maturitas. 2020;136:42-8. doi: 10.1016/j.maturitas.2020.04.008.

20. Wang $\mathrm{M}$, Gong WW, Hu RY, Wang H, Guo Y, Bian Z, et al. Age at natural menopause and associated factors in adult women: Findings from the China Kadoorie Biobank study in Zhejiang rural area. PLoS One. 2018;13:e0195658. doi: 10.1371/journal.pone.0195658.

21. Bouxsein ML, Eastell R, Lui LY, Wu LA, de Papp AE, Grauer A, et al. Change in Bone Density and Reduction in Fracture Risk: A Meta-Regression of Published Trials. J Bone Miner Res. 2019;34:63242. doi: $10.1002 / \mathrm{jbmr} .3641$.

22. Yun $Z$, Cuiping $M$, Weimin $Y$, Xiaoxiang $Z$, editors. Application of cell engineering of herbal Medicine treating bone resorption of osteoclasts. 2005 IEEE Engineering in Medicine and Biology 27th Annual Conference; 2005 17-18 Jan. 2006.

\section{Figures}

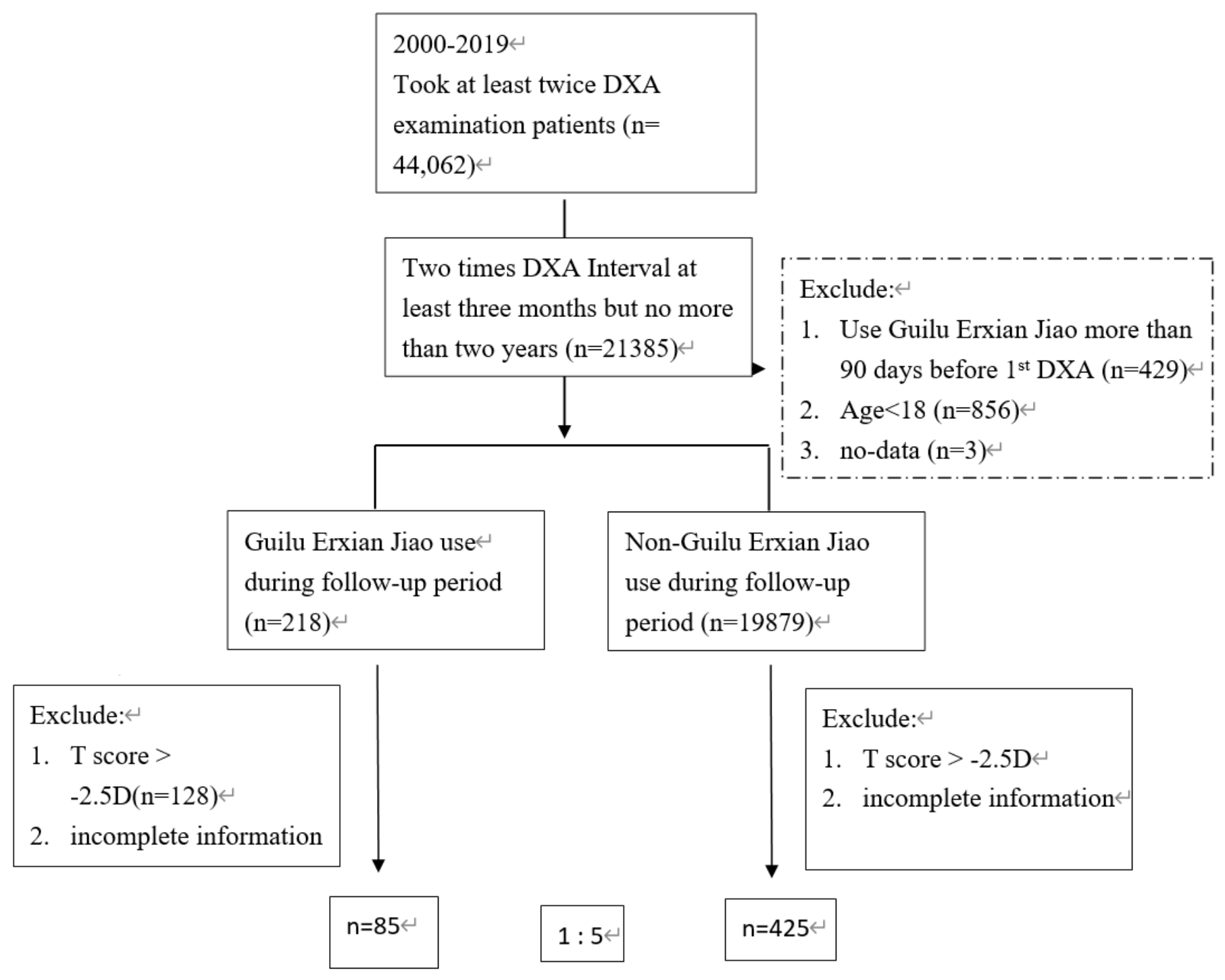

Figure 1 


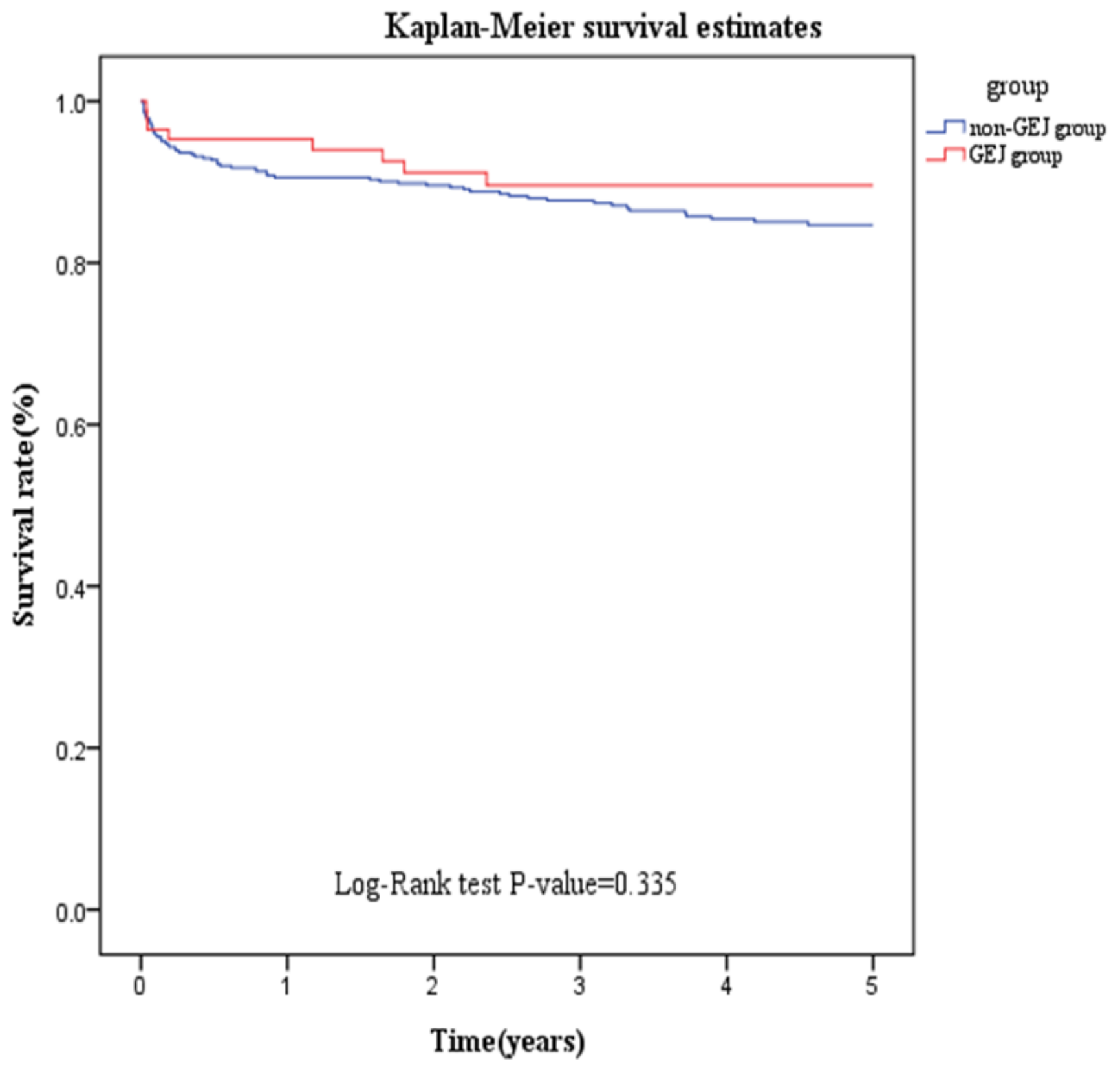

Figure 2

Comparison of Kaplan-Meier curve of the difference between the Guilu Erxian Jiao user and non-Guilu Erxian Jiao user groups in the development of fracture. GEJ, Guilu Erxian Jiao. 


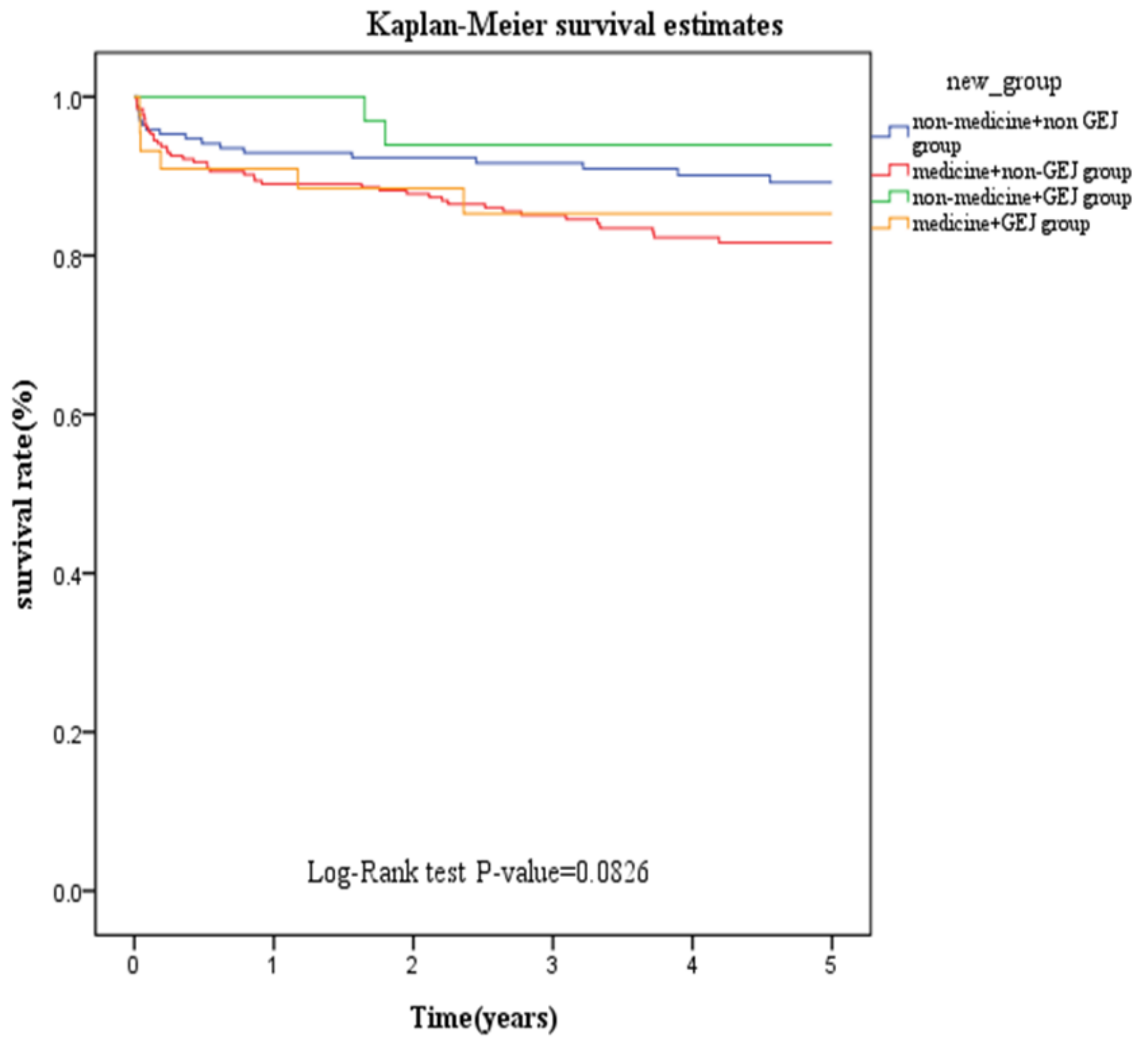

Figure 3

Comparison of KaplanMeier failureestimates offracture onset among the four groups. GEJ, Guilu Erxian Jiao. 


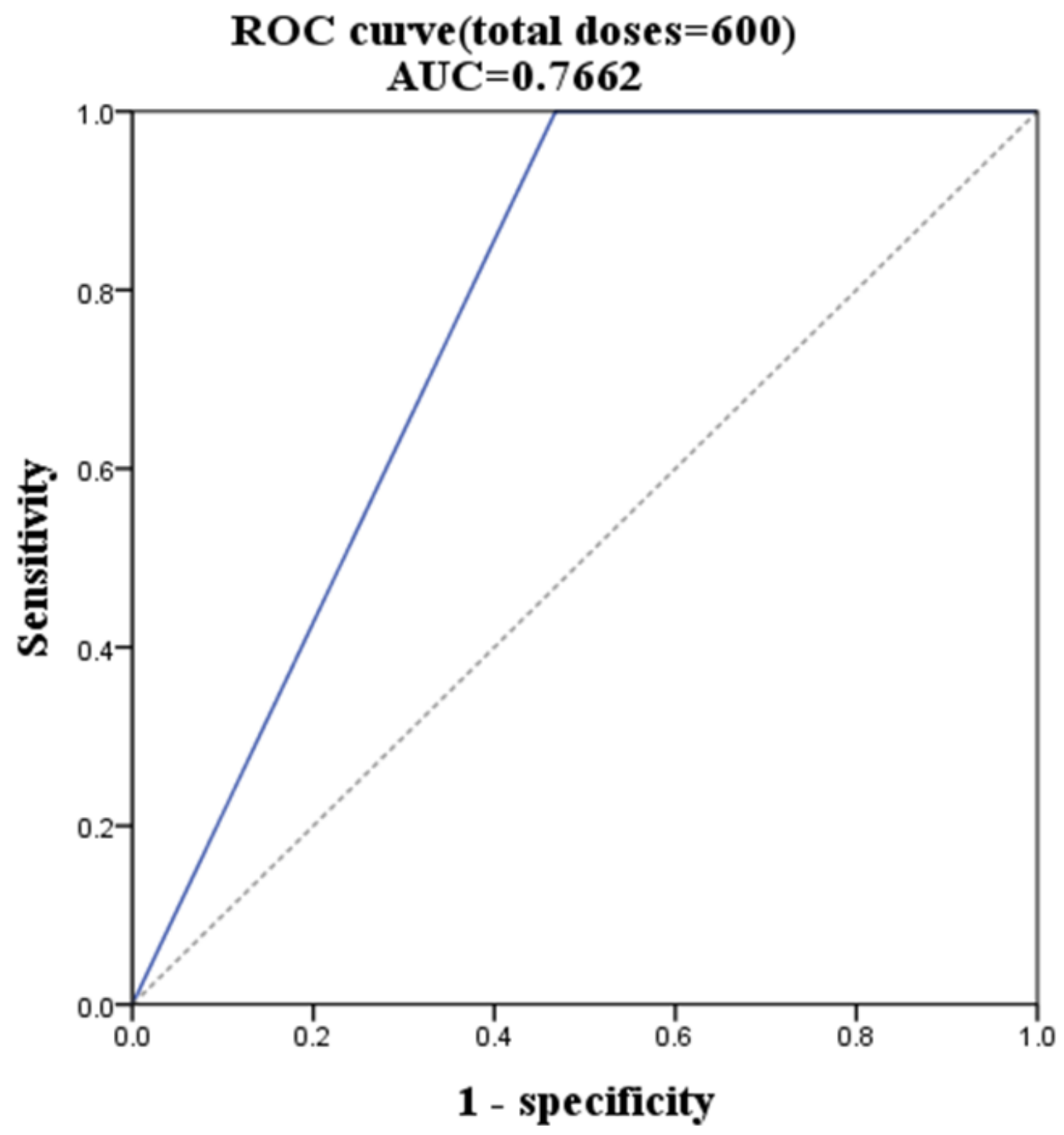

Figure 4

ROC curve according to total dose of Guilu Erxian Jiao use amount (dose multiplied use days) 


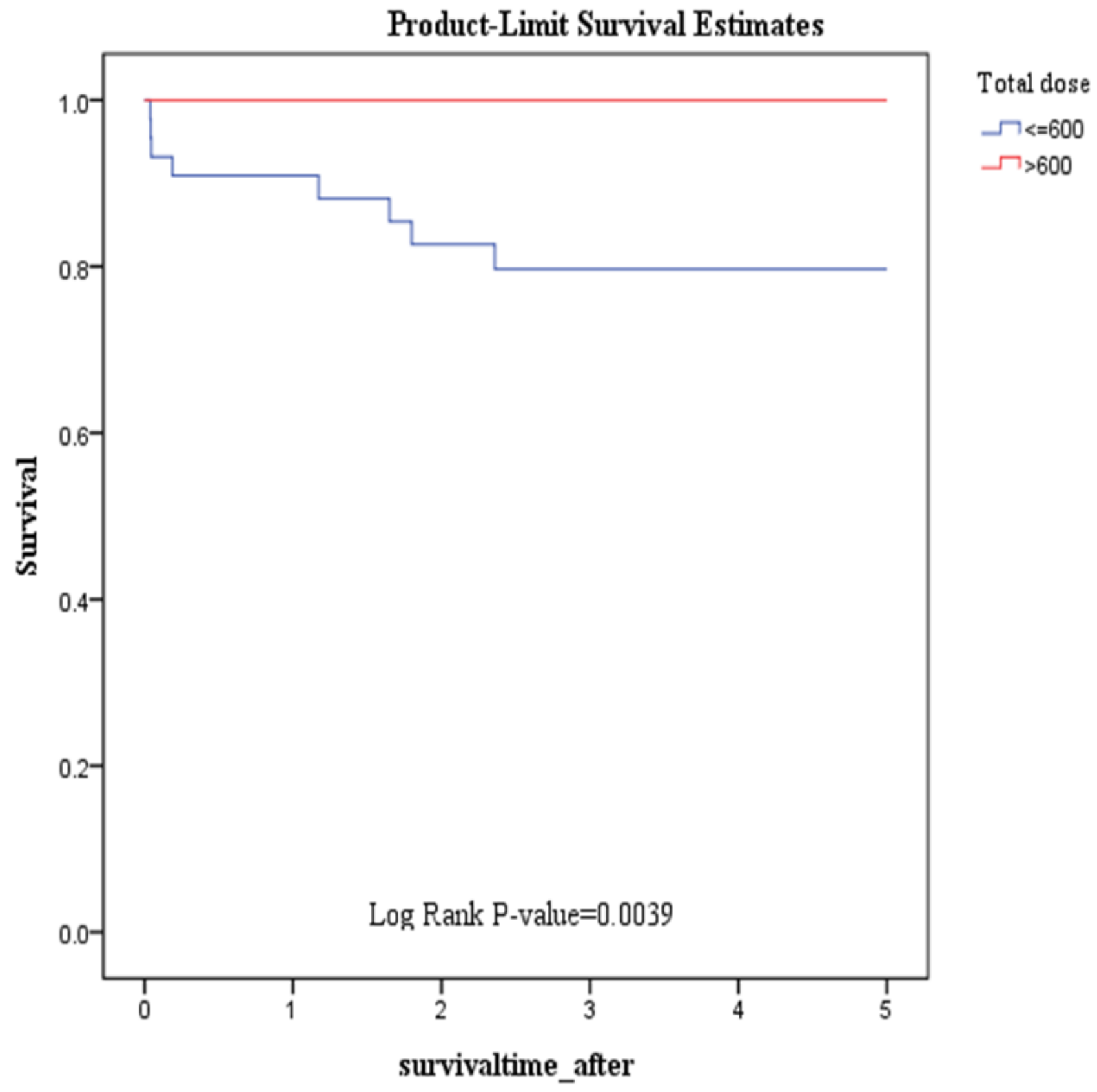

Figure 5

Kaplan-Meier curve of the difference between the high total dose Guilu Erxian Jiao(>600 pills), the low total dose Guilu Erxian Jiao(<=600 pills) in the development of fracture.

\section{Supplementary Files}

This is a list of supplementary files associated with this preprint. Click to download.

- Additionalfile1.pdf 